\title{
A ROLE FOR YOUTUBE IN TELEREHABILITATION
}

\author{
M. Hunter manasco, PhD, Nicholas BARONE, BS, \\ AMANDA BROWN, BS \\ DEPARTMENT OF SPEECH-LANGUAGE PATHOLOGY, Misericordia UNIVERSITY
}

\section{ABSTRACT}

YouTube (http://youtube.com) is a free video sharing website that allows users to post and view videos. Although there are definite limitations in the applicability of this website to telerehabilitation, the YouTube technology offers potential uses that should not be overlooked. For example, some types of therapy, such as errorless learning therapy for certain language and cognitive deficits can be provided remotely via YouTube. In addition, the website's social networking capabilities, via the asynchronous posting of comments and videos in response to posted videos, enables individuals to gain valuable emotional support by communicating with others with similar health and rehabilitation challenges. This article addresses the benefits and limitations of YouTube in the context of telerehabilitation and reports patient feedback on errorless learning therapy for aphasia delivered via videos posted on YouTube.

Key words: telerehabilitation, speech-language therapy, YouTube, aphasia

\section{INTRODUCTION}

YouTube $\AA$ (http://youtube.com) is a video sharing website that allows anyone to post videos online for viewing. This website is one of the most popular on the Internet. Wesch (2008) reported that in 2008, there were 78.3 million videos available on YouTube. Wesch further estimated that that it would require 412.3 years for a person to watch all those videos. Users can view videos posted on the website and comment on, rate, and make video responses to posted videos. At first glance, the applicability of YouTube to telerehabilitation in general, and speech-language therapy in specific, seems extremely limited and possibly nonexistent. However, there are possible applications and benefits of YouTube in telerehabilitation that need to be considered to maximize patients' levels of recovery.

\section{YOUTUBE AND REHABILITATION AT PRESENT}

When the term rehabilitation therapy is searched on YouTube a multitude of videos will appear. Specifically, on April 4, 2010, a search of the word rehabilitation produced 12,800 results. Many of these videos were created post- morbidly by stroke or trauma victims in an attempt to share the therapy exercises they learned during rehabilitation with others with similar problems.

There are also many videos posted by physical therapists and speech-language pathologists to provide homework instructions to their patients on how to help themselves recover from injury. Homework therapy is nothing new in rehabilitation and is often an important factor in maximizing a patient's level of recovery. However, the utility of many of these videos to persons with no contact with a clinician is questionable at best and dangerous at worst.

\section{LIMITATIONS FOR TELEREHABILITATION}

The limitations of YouTube's possible applicability in telerehabilitation are significant. The patient must possess a computer with Internet access at home and be able to navigate to the website to retrieve posted videos. This may be problematic because many rehabilitation patients are of a lower socioeconomic status and may possess cognitive and language deficits following trauma or stroke that keep them from being able to operate a computer.

Placing an individual with cognitive impairments such as decreased problem solving and increased impulsivity online may be problematic. Burton (2008) reported that there are patient help pages on YouTube that have been paid for by pharmaceutical companies which advocate the use of certain drugs. Also, when physical therapy is searched on YouTube the viewer is soon directed to videos posted by companies hoping to sell products meant to ease back pain and other general ailments. This 
scenario brings into question the possibility of strongly biased information regarding certain health conditions being present on YouTube. Patients could also access therapy videos on YouTube that may be inappropriate or dangerous for their deficits. Although patients could easily be warned against these threats and practices, many patients will have cognitive impairments that will limit the efficacy of warnings.

Another limitation is that, unlike video conferencing, YouTube allows no direct and real time interaction between the patient and the clinician. Most rehabilitation therapies, including speech and language therapy are traditionally highly dependent on immediate and real time feedback from the clinician as well as adjustments made by the clinician in ongoing therapy tasks and goals.

\section{ADDRESSING THESE LIMITATIONS}

The lack of computers with Internet access in households is a concern for anyone considering using YouTube for telerehabilitation purposes. However, the U.S. Census Bureau reports that as of 2009, 68.7 percent of all households in the United States have a computer with Internet access. These numbers suggest that a lack of the necessary technology may not be a concern for a majority of patients.

The presence of cognitive deficits that may inhibit the patient's ability to use a computer to access the Internet is an obvious concern, especially for speechlanguage pathologists involved in the rehabilitation of cognition. However, because YouTube is such a popular website, there may be some premorbid familiarity and user knowledge of the website among the population of rehabilitation patients which may reduce this concern as a limitation. Even a lack of familiarity with the website in patients may be turned to therapeutic benefit if used in cognitive therapies to address new learning or problem solving deficits.

Certain simple steps can be taken to reduce exposing cognitively impaired patients to faulty or inappropriate health information, online predators, and deceitful marketing ploys. It may be necessary for a caregiver to monitor the patient while online. Also, Microsoft's Windows is the most popular operating system for PCs and features parental controls. The parental controls built into Windows allow parents to restrict the online behavior of their children to certain websites, games, or programs. These parental controls may also be used by caregivers to ensure the protection of their cognitively impaired loved ones when working online. Clinicians can also limit which of their videos are accessible by patients by creating private channels on YouTube. In this way a clinician can prevent a patient from accessing videos that may be inappropriate or dangerous for the patient.

Perhaps the most significant limitation is that YouTube can only be used to play videos previously uploaded to the website and thereby provides no direct and real time interaction between clinician and patient. There is no practical way to use YouTube that compensates for the lack of simultaneous visual interaction between the therapist and patient. However, the use of a telephone can create a real time audio connection between therapist and patient through which clinicians' instructions and feedback to the patient can be given while the patient is viewing and working through a video created by the clinician. This audio connection between the patient and the clinician can be established via a computer based phone call or by simply using the patient's speaker phone capability present on their home telephone. Most telephones are now sold with speaker phones built in, even though many individuals may not be aware of the capability. In the above described scenario the clinician will only have access to the patient's verbal narrative of their performance on the task presented on the video. This situation is very inferior to a video conferencing setup where the patient and clinician may interact visually as well as verbally. However, if a patient and therapist have been through many face-to-face sessions the clinician may have a very good idea of what is happening as the patient progresses through the video and the clinician may not be entirely dependent on visual information regarding the patient's performance. Also, using the above scenario would require only minimal training, and can possibly be implemented by purchasing no additional equipment, software, or services. This lack of additional cost may make the delivery of certain services using YouTube more feasible for some patients who are unwilling or unable to travel to receive therapy or use videoconferencing for therapy. In addition, certain therapies may be more readily delivered via YouTube than others.

\section{YOUTUBE AND ERRORLESS THERAPY FOR APHASIA}

Within the realm of therapy for aphasia and cognition, the therapy approach called errorless learning seems particularly well matched for delivery via YouTube. Researchers who have examined errorless learning approaches have produced results suggesting that errorless therapies for anomia and memory deficits are effective in remediation of these deficits (Fillingham, Sage, Lamden, \& Ralph, 2006; McKissock \& Ward, 2007; Mulholland, Donoghue, Meenagh, \& Rushe, 2008; Ruis \& Kessels, 2005). Errorless therapies capitalize on intact procedural memory for rehabilitation of anomia and memory deficits, instead of relying on impaired declarative memory abilities. These therapies are also effective because they focus on reducing the practicing and reinforcement of error production.

The primary basis of errorless learning is that the stimuli delivered are well within the ability level of the patient to 
successfully accomplish therapy tasks with no trial and error behaviors. Errorless therapy stimuli are selected to facilitate patient success and minimize patient failure. If therapy stimuli are presented in this way then it can be reasonably argued that the role of the therapist in deliverance of this type of therapy is minimal. If there is no possibility of patient failure on a therapy task then there is no need for the clinician to be scaffolding the patient towards success, delivering corrective feedback, or adjusting the therapy tasks to facilitate success. This reduces or eliminates the need of the clinician to be on hand for the patient to complete errorless activities. The clinician will still be required to choose appropriate therapy tasks, set the therapy stimuli at the appropriate difficulty level, and track patient progress over time. However, the bulk of this therapy could be delivered via a video of errorless therapy stimuli created by the clinician and uploaded to YouTube for the patient to access on their own time and complete independently at home or wherever they have access to a computer with an Internet connection.

This model was piloted by the authors to test real world feasibility and to provide increased amounts of therapy to a patient with moderate level of anomia following a cerebrovascular accident. Three videos were created and posted on YouTube. Each video presented six common objects to the patient twice. Each object was presented for fifteen seconds. Objects and their names were presented visually for the patient to produce. To ensure patient success and minimize failure a verbal semantic cue was presented as well (i.e., You brush your teeth with a...). The patient completed each of the three videos twice in each condition. The videos were presented in two conditions. During the first condition the patient worked through the videos while receiving verbal feedback regarding his performance over a speakerphone from a clinician at another location. During the second condition the patient completed the videos independently in a room alone. The patient displayed $100 \%$ success on each video every time it was presented, which is desirable in errorless therapy. Afterwards, the patient was questioned regarding his experience and impressions regarding the therapy and the delivery model.

This patient's responses to this pilot were very positive. When asked what his general impression of this attempt was he responded, "I think it was a good idea." When asked if he would independently work through these videos at home he responded, "I would probably do it." Given the low difficulty level of the pilot stimuli the primary researcher also asked the patient if he found the low difficulty level of the stimuli boring or tedious. The patient responded that he didn't find it tedious and that he thought many individuals with severe aphasia deficits could probably benefit. In the quote below the patient indicates his belief that this kind of activity allows individuals to take responsibility for their own rehabilitation and that by being able to complete the tasks without a therapist the patient will be empowered.

Patient: "I think... Because at least you are thinking you are making progress, you know? Somebody with a stroke... that would be a real... Because they could almost think that they were doing it themselves, and that gives them the feeling 'I am'."

In the next utterance transcribed below the patient indicates his belief that the errorless therapy would make individuals with aphasia feel good about themselves and make them feel like they were making progress.

Patient: "It makes it so that the client feels like they are making progress too... You know? And maybe they're not... but it seems like it though. Then they think... they'll do. But I think it is a good idea."

When asked if he felt that he needed the clinician's verbal feedback provided to him via the speakerphone he responded, "I didn't have the need for her [the clinician] but someone else may."

This brief and qualitative investigation of this method is very limited. Nonetheless, the responses that the patient gave are very insightful and provide a strong rationale for future exploration of this YouTube delivery method of errorless therapy approaches in aphasia.

\section{SOCIAL NETWORKING ON YOUTUBE}

Negative emotional reactions to stroke and trauma such as depression are common (Chapey, 2008) and often go untreated (Clark \& Smith, 1998; Masand \& Chaudhaury, 1994: as cited in Chapey [2008]). Leaving negative emotional reactions unaddressed is known to reduce progress in therapy (Masand \& Chaudhaury, 1994: as cited in Chapey [2008]). Meeting and communicating with others with problems similar to their own is often an effective coping mechanism for individuals with health problems and can provide an important emotional outlet. However, many individuals who have experienced severe trauma or stroke lack the physical ability to attend group therapy or support activities in person.

YouTube is able to play a unique role in addressing the emotional needs of patients during and after rehabilitation in remote areas or areas that have no possibility of meeting others with similar problems. It is capable of performing this function by providing networking opportunities between individuals with similar problems. Below every video posted on YouTube is a discussion board in which people are able to comment on the video or ask questions. Users can also email questions to 
each other through the YouTube website without publicly posting their comments online. Individuals with health problems, and sometimes very rare conditions, often post videos of themselves on YouTube describing their problem and asking for support and advice. Very often, they receive a great deal of online support in response to their requests. For example, a young woman with a rare neurological syndrome recently posted a video asking if anyone knew any therapy treatment options for her difficulties with facial movement. This young woman lived outside of the United States, where she stated that she knew of no other individuals with her disorder. Despite her isolated geographical location she received and refereed a lively discussion of treatment options for her problem in response to this video. Contributions to this discussion were offered by parents of children with the same neurological syndrome, other individuals with the syndrome, as well as students and other professionals from around the world. In another example, a young man, also with a neurological syndrome, posted a video of himself describing his condition and his problems with physical movement. He extended an open invitation for others to contact him. Within less than two years this video has been viewed over 3,000 times and the user has received supportive responses from many viewers with similar difficulties from many different countries. One individual posted a video response and spoke of the value of YouTube in connecting individuals with rare diseases and health problems. She stated that it was nice to meet others with the same problems on YouTube and that she had never met, in person, another individual with the same syndrome.

In this same fashion, YouTube is clearly able to offer individuals with common health problems the ability to network and communicate with others around the globe for free. For individuals in rehabilitation, this additional emotional support may help support their efforts in therapy and increase their overall level of recovery.

\section{CONCLUSIONS}

Despite the limitations, the benefits of YouTube as a resource for rehabilitation are significant. Remote implementation of many therapies via YouTube may be awkward and inefficient. However, there is a possible niche for YouTube in the deliverance of a certain level of errorless therapy, at least in the rehabilitation of anomia and memory deficits, as well as providing emotional support by allowing patients to meet and communicate with others with similar problems. Given that YouTube is an entirely free resource and many patients will have home access, it is logical that this resource should be exploited as fully as possible to maximize the recovery of those in need.

\section{AUTHORS' NOTE}

Special thanks to our patients for granting formal permission to include their quotes.

\section{REFERENCES:}

Chapey, R. (2008). Language intervention strategies in aphasia and related neurogenic communication disorders. Philadelphia, PA; Lippincott, Williams and Wilkins.

Clark, M.S., \& Smith, D.S. (1998). The effects of depression and abnormal illness behaviour on outcome following rehabilitation from stroke. Clinical Rehabilitation, 12, 73-80.

Fillingham, J.K., Sage, K., Lambon Ralph, M.A. (2006). The treatment of anomia using errorless learning. Neuropsychological Rehabilitation, 16(2), 129-154.

McKissock, S., \& Ward, J. (2007). Do errors matter? Errorless and errorful learning in anomic picture naming. Neuropsychological Rehabilitation, 17(3), 355-373.

Mulholland, C.C., Donoghue, D., Meenagh, C., \& Rushe, T.M. (2008). Errorless learning and memory performance in schizophrenia. Psychiatry Research, 159, 180-188.

Ruis, C., \& Kessels, R.P. (2005). Effects of errorless and errorful face-name associative learning in moderate to severe dementia. Aging Clinical and Experimental Research, 17(6), 514-517.

Wesch, M. (2008). YouTube Statistics. Retrieved January 13, 2010, from Digital Ethnography at Kansas State University Web site: http://mediatedcultures.net/ ksudigg/?p=163 